\title{
HÁBITOS DE CONSUMO DE SUPLEMENTO DE VITAMINA C DURANTE A PANDEMIA DO COVID-19: BENEFÍCIOS, RISCOS E O PAPEL DA ASSISTÊNCIA FARMACÊUTICA NO USO RACIONAL
}

\section{Agatha de Amorim Laurindo', Jessica da Silva Reis ${ }^{1}$, Leandro Giorgetti ${ }^{2, A}$}

${ }^{A}$ Acadêmica do curso de farmácia da Universidade Anhembi Morumbi - São Paulo - Brasil.

${ }^{2}$ Orientador e docente do curso de farmácia da Universidade Anhembi Morumbi - São Paulo - Brasil.

\section{RESUMO}

Com o início da pandemia da doença coronavírus 2019 (COVID-19), inúmeros regimes de tratamentos têm sido empregados. Ainda que não existam dados de ensaios clínicos que apoiem qualquer tratamento profilático, além das vacinas, outros medicamentos off-label estão sendo empregados, bem como a suplementação de vitamina C. Para o presente artigo, foi realizado um estudo a fim de entender hábitos de consumo de suplementos vitamínicos orais, sobretudo a vitamina $\mathrm{C}$, no período de pandemia de COVID-19, analisando de que maneira e com qual frequência o consumo ocorria e os motivos que levaram ao início do tratamento. Para coletar respostas, foram disponibilizadas, de maneira on-line, 12 perguntas codependentes utilizando a plataforma Microsoft Forms ${ }^{\circledR}$. O formulário obteve 1305 respostas elegíveis. As idades dos entrevistados variavam entre 18 anos e mais de 46 anos, sendo $70 \%$ ( $n=916)$ do gênero feminino. Dos participantes, 1010 citaram não ter contraído COVID-19. Desses, 39,7\% ( $n=400)$ faziam uso de vitamina C isolada ou concomitantemente com vitamina D. De 296 participantes que alegaram ter tido COVID-19, 42,91\% ( $n=127)$ faziam uso de vitamina $C$ isolada ou em conjunto com vitamina $D$, enquanto $48,3 \%(n=143)$ não utilizavam nenhum suplemento vitamínico. Dos entrevistados, $28,3 \%(n=189)$ apontaram ter iniciado o uso da vitamina $C$ devido a pandemia. Além de interações medicamentosas, o consumo da vitamina $C$ em quantidades maiores que a dose diária recomendada, pode acarretar eventos adversos gastrintestinais. Dos participantes, $43,1 \%(n=563)$ responderam acreditar que o consumo de vitaminas sem acompanhamento médico ou farmacêutico não oferece prejuízo a saúde, reforçando a substancialidade da conscientização no uso racional de suplementos vitamínicos, sobretudo pelos riscos que o consumo sem orientação médica ou farmacêutica pode trazer à saúde.

Palavras-chave: COVID-19; Suplementação; Vitamina C

\section{ABSTRACT}

With the onset of the coronavirus disease (COVID-19) pandemic, several treatment regimens have been employed. Although, there are no clinical trial data to support any prophylactic treatment in besides vaccines, some off-label drugs are being used, as well as vitamin $\mathrm{C}$ supplementation. This study was carried out in order to understand habits of consumption of oral vitamin 
supplements, especially vitamin C during the COVID-19 pandemic period, to analyze how and how often the consumption occurred and the reasons that led to the start of treatment. To collect answers online, 12 codependent questions were made available using the Microsoft Forms $\AA$ platform. Were received 1305 eligible answers. Participants' ages ranged from 18 years to over 46 years, with $70 \%$ ( $n=916$ ) being female. Of the participants, 1010 mentioned not having been infected by COVID-19. Of these, $39.7 \%(n=400)$ were using pure vitamin C or concomitantly with vitamin D. Of 296 participants who claimed to have had COVID-19, 42.91\% $(n=127)$ were using pure vitamin $C$ or together with vitamin $D$, while $48.3 \%(n=143)$ did not use any vitamin supplement. Of the respondents, $28.3 \%(n=189)$ indicated that they had started using vitamin $\mathrm{C}$ due to the pandemic. In addition to drug interactions, the consumption of vitamin $\mathrm{C}$ in amounts greater than the recommended daily dose can lead to adverse gastrointestinal events. Of the participants, $43.1 \%(n=563)$ responded that they believed that the consumption of vitamins without medical or pharmaceutical supervision does not harm their health, reinforcing the substantiality of awareness in the rational use of vitamin supplements, especially due to the risks that consumption without guidance medical or pharmaceutical can bring health.

Key words: COVID-19; Supplementation; Vitamin C

\section{INTRODUÇÃO}

Com o início da pandemia da doença coronavírus 2019 (COVID-19) causada pelo vírus SARS-CoV-2, inúmeros regimes de tratamentos têm sido empregados [1]. Atualmente, nenhum medicamento ainda se mostrou eficaz, salvo o remdesivir, aprovado pela Food and Drug Administration (FDA) para o tratamento de COVID-19 [2,3]. Ainda que não existam dados de ensaios clínicos que apoiem qualquer tratamento profilático [4], além das vacinas, outros medicamentos off-label estão sendo empregados, bem como a suplementação de vitamina C [2].

A vitamina $\mathrm{C}$, também conhecida como ácido ascórbico, L-ácido ascórbico, ascorbato, é um micronutriente essencial, ou seja, não é sintetizada em seres humanos devido à perda de uma enzima chave na via biossintética e precisa ser adquirida através da dieta [5]. É um potente antioxidante, em razão da sua capacidade de doar elétrons. Lipídeos, carboidratos, e outras importantes biomoléculas são protegidas do dano oxidativo proveniente do metabolismo natural celular e de toxinas provenientes do meio ambiente [6].

Com o advento da medicina ortomolecular por Linus Pauling foi possível correlacionar o uso da vitamina C com a manutenção do sistema imunológico, e isso incentivou a utilização da vitamina com a finalidade de prevenir e reduzir a duração do resfriado comum [7]. Desde aquela época, a vitamina C e seus efeitos protetores têm sido estudados no resfriado comum. Vários estudos demonstraram que a suplementação de vitamina $C$ estimula o sistema imunológico ao aumentar a proliferação de células $\mathrm{T}$, responsáveis por lisar alvos infectados, produzindo quantidade considerável de citocinas, e auxiliando as células $\mathrm{B}$ a sintetizar imunoglobulinas para regular as reações inflamatórias, no decorrer da infecção [8].

O coronavírus SARS-CoV-2 consegue se replicar no trato respiratório inferior, causando infecção, e sendo capaz de desencadear pneumonia [9]. Neste âmbito, estudos sugeriram que os agentes antioxidantes, sobretudo, a vitamina $\mathrm{C}$ apresentam um papel importante em algumas infecções virais [10]. Três ensaios clínicos controlados em seres humanos relataram uma incidência significativamente menor de pneumonia em grupos suplementados com ácido ascórbico, sugerindo que, sob certas condições, a vitamina $\mathrm{C}$ pode prevenir a suscetibilidade a infecções do trato respiratório inferior [11].

Além de desempenhar vários efeitos benéficos no sistema imunológico inato e adaptativo [6], a vitamina C também está envolvida contra Doenças Crônicas Não Transmissíveis (DCNT) como obesidade [12], diabetes mellitus [13], hipertensão arterial [14], doenças cardiovasculares [15], doenças renais [16] e câncer [17], associadas à seriedade e mortalidade da doença COVID-19 $[18,19]$.

Dessa forma, o objetivo do estudo foi estabelecer os hábitos de consumo de vitamina C durante a pandemia da COVID-19, apontando seus benefícios e riscos, bem como o papel da assistência farmacêutica no uso racional desse suplemento.

\section{MATERIAIS E MÉTODOS}

Na presente investigação, foi realizado um estudo qualitativo e quantitativo, com caráter descritivo e transversal, a fim de entender hábitos de consumo de suplementos vitamínicos orais, sobretudo a vitamina C no período de pandemia de COVID-19, analisar de que maneira e com qual frequência o consumo ocorria e os motivos que levaram ao início do tratamento.

Para coletar respostas foram disponibilizadas 12 perguntas codependentes e que seguiam uma sequência lógica, utilizando a plataforma Microsoft Forms $\AA$, seguindo os preceitos de ética dados na Resolução N466/2012. Somente após aprovação do Comitê de Ética em Pesquisa da Universidade Anhembi-Morumbi, conferido pelo parecer 4.710 .749 em 13 de maio de 2021, o formulário foi compartilhado e a coleta de respostas iniciada. $O$ questionário só foi respondido por aqueles que autorizaram a sequência e sua participação.

Para construção da análise estatística e quantificação de resultados, foi utilizada a planilha em Microsoft Excel ${ }^{\circ}$ gerada de maneira automática pela plataforma de coleta, documento esse 
também utilizado para criação de estatísticas visuais tabeladas e gráficos que contribuíram para o artigo.

\section{RESULTADOS E DISCUSSÃO}

\section{Perfil geral dos participantes}

O formulário ficou disponível entre 14 de maio a 21 de junho de 2021 e gerou 1321 respostas. Dos participantes, 16 não concordaram com os termos propostos, por isso não tiveram nenhuma outra informação coletada. Das 1305 participações elegíveis, $70 \%(n=916)$ eram do sexo feminino, $30 \%(n=387)$ eram do sexo masculino e $0,1 \%(n=2)$ participantes possuíam outro gênero (não informado).

As idades dos entrevistados variavam entre 18 anos e mais de 46 anos, conforme apresentado no Gráfico 1.

Gráfico 1: Perfil dos entrevistados, distribuição de idade entre os gêneros feminino e masculino.

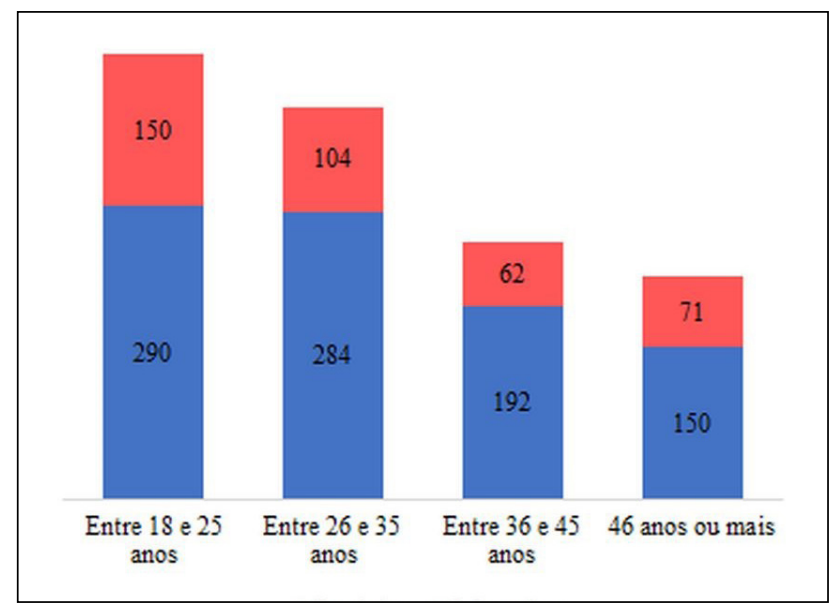

Fonte: Autores.

\section{Frequência geral do uso de vitaminas}

Dos participantes entrevistados, 637 participantes apontaram não fazer utilização de nenhum suplemento, enquanto, 668 alegaram fazer alguma suplementação vitamínica. Desses, $34,6 \%(n=231)$ com uso exclusivo de vitamina C, 44\% $(n=294)$ informaram fazer uso de suplementação concomitante de vitamina C e D, e $21,4 \%$ ( $n=143)$ disseram fazer uso de outras vitaminas, como demonstrado no Gráfico 2.

Como mostrado na Tabela 1, a maior parte dos participantes consomem a vitamina $C$ pura ou em conjunto com a vitamina $D$ uma vez ao dia. Um comportamento semelhante foi encontrado em um estudo realizado por Paiva et al. [20], em que a maioria dos entrevistados também relatou fazer uso de suplementos diariamente.

Pandemia de CoVID-19: Vitamina C e assistência farmacêutica
Gráfico 2: Perfil de distribuição de consumo de suplementos vitamínicos entre os gêneros feminino e masculino.

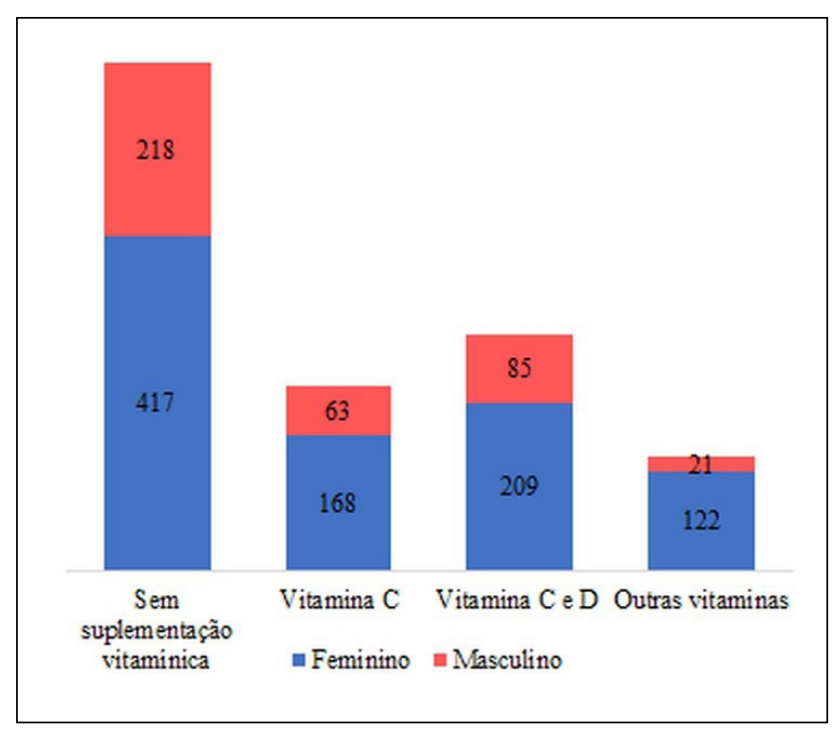

Fonte: Autores.

De acordo com Mendes et al. [21], o isolamento social no Brasil causado pela pandemia de COVID-19 levou as pessoas a buscarem uma alimentação mais saudável, com foco em alimentos e nutrientes que atuam e modulam o sistema imunológico. Além disso, um estudo de infodemiologia utilizando dados do Google Trends evidenciou um aumento significativo de pesquisas no buscador com os termos "vitamina C", "imunidade" e "COVID-19" em março de 2020 , se comparado ao mesmo período nos anos anteriores [22].

Similarmente, outra vitamina que tem sido amplamente utilizada durante a pandemia da doença COVID-19 é a vitamina D. Existem diversos estudos em andamento que visam avaliar o potencial benefício da coadministração da vitamina C e D. Em países da Europa, a deficiência da vitamina $D$ foi associada a maior mortalidade em casos de infecção pelo vírus SARS-CoV2 [23]. Por isso, dentre as opções de resposta da presente pesquisa, essa era uma das alternativas possíveis.

Dos participantes, 1010 citaram não ter contraído COVID-19. Desses, $39,7 \% \quad(n=400)$ faziam uso de vitamina $C$ pura ou concomitantemente com vitamina D. De 296 participantes que alegaram ter tido COVID-19, 42,9\% $(n=127)$ faziam uso de vitamina C pura ou em conjunto com vitamina $D$, enquanto $48,3 \% \quad(n=143)$ não utilizavam nenhum suplemento vitamínico. Os outros $8,8 \%$ $(n=26)$ consumiam outras vitaminas, conforme demonstrado no Gráfico 3.

Segundo Sudre et al. [24], a gravidade da sintomatologia da COVID-19 está subdividida em 6 classes, conforme exposto na Tabela 2. Verificou-se que 12 pessoas apresentaram o quadro mais severo de sintomas, dessas, $50 \%(n=6)$ não fazem suplementação vitamínica.

Dentre os participantes que contraíram COVID-19, 40 foram assintomáticos, desses, $52,5 \%(n=21)$ faziam uso da vitamina $C$ pura ou em conjunto com vitamina $\mathrm{D}$, conforme o Gráfico 4 . 
Tabela 1: Frequência de utilização da vitamina $C$ dos entrevistados.

\begin{tabular}{|c|c|c|c|c|c|c|}
\hline & $\begin{array}{c}\text { Mais de uma } \\
\text { vez ao dia } \\
\text { (1x ao dia) }\end{array}$ & $\begin{array}{c}\text { Diariamente } \\
\text { semana }\end{array}$ & $\begin{array}{c}\text { Três ou mais } \\
\text { vezes por } \\
\text { por semana }\end{array}$ & $\begin{array}{c}\text { Unas vezes } \\
\text { semana (mas não } \\
\text { diariamente) }\end{array}$ & $\begin{array}{c}\text { Quinzenalmente } \\
\text { por mês }\end{array}$ \\
\hline Vitamina C & 11 & 154 & 22 & 6 & 18 & 16 \\
\hline Vitamina C e D & 12 & 232 & 14 & 9 & 4 \\
\hline Outras & 3 & 56 & 72 & 4 & 0 & 8 \\
\hline
\end{tabular}

Fonte: Autores.

Gráfico 3: Comparativo dos entrevistados que alegaram ter contraído ou não COVID-19.

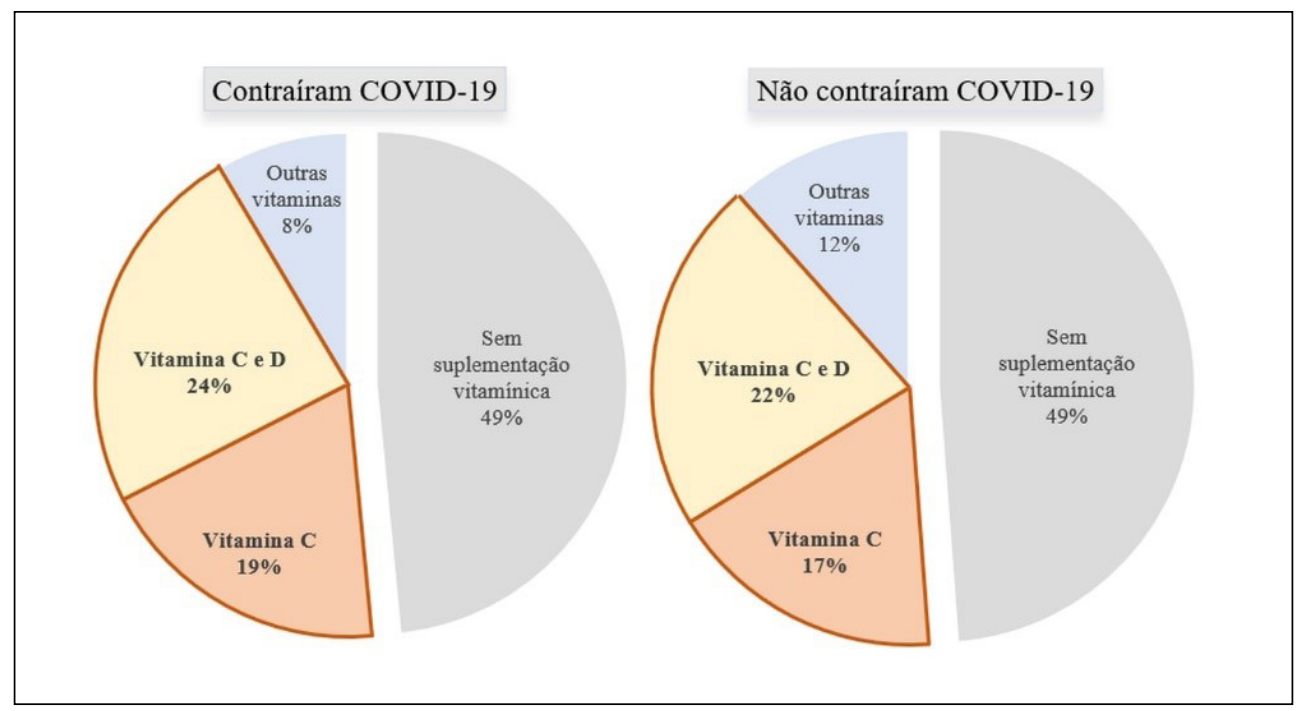

Fonte: Autores.

Tabela 2: Classificação da sintomatologia da COVID-19 e consumo de suplementos vitamínicos dos entrevistados.

\begin{tabular}{|c|c|c|c|c|}
\hline & $\begin{array}{l}\text { Não faço suplementação das } \\
\text { vitaminas abaixo informadas }\end{array}$ & Vitamina C & Vitamina C e D & $\begin{array}{c}\text { Outras } \\
\text { vitaminas }\end{array}$ \\
\hline Assintomático & 11 & 13 & 8 & 8 \\
\hline $\begin{array}{l}\text { Quadro semelhante à gripe, com febre: Dor de } \\
\text { cabeça, perda de olfato, tosse, dor de garganta, } \\
\text { rouquidão, perda de apetite, febre. }\end{array}$ & 47 & 18 & 13 & 2 \\
\hline $\begin{array}{c}\text { Quadro semelhante à gripe, sem febre: Dor de } \\
\text { cabeça, perda de olfato, dor muscular, tosse, dor de } \\
\text { garganta, dor no peito, sem febre }\end{array}$ & 45 & 16 & 22 & 8 \\
\hline $\begin{array}{c}\text { Quadro gastrointestinal: Dor de cabeça, perda } \\
\text { de olfato, dor de garganta, dor no peito, perda de } \\
\text { apetite, diarreia, sem tosse }\end{array}$ & 11 & 2 & 5 & 5 \\
\hline $\begin{array}{c}\text { Quadro severo nível } 1 \text { (fadiga): Dor de cabeça, } \\
\text { perda de olfato, tosse, febre, rouquidão, dor no } \\
\text { peito, fadiga }\end{array}$ & 11 & 4 & 10 & 1 \\
\hline
\end{tabular}




\begin{tabular}{|c|c|c|c|c|}
\hline $\begin{array}{c}\text { Quadro severo nível 2 (confusão mental): Dor de } \\
\text { cabeça, perda de olfato, perda de apetite, tosse, } \\
\text { rouquidão, dor de garganta, dor no peito, dor } \\
\text { muscular, febre, fadiga, confusão mental }\end{array}$ & 12 & 3 & 9 & 0 \\
\hline $\begin{array}{c}\text { Quadro severo nível 3 (falta de ar): Dor de } \\
\text { cabeça, perda de olfato, perda de apetite, tosse, } \\
\text { rouquidão, febre, dor de garganta, dor no peito, } \\
\text { fadiga, confusão mental, dor muscular, diarreia, dor } \\
\text { abdominal, dificuldade para respirar. }\end{array}$ & 6 & 0 & 4 & 2 \\
\hline
\end{tabular}

Fonte: Autores.

Gráfico 4: Perfil de consumo de suplementos vitamínicos dos entrevistados que contraíram COVID-19 e foram assintomáticos.

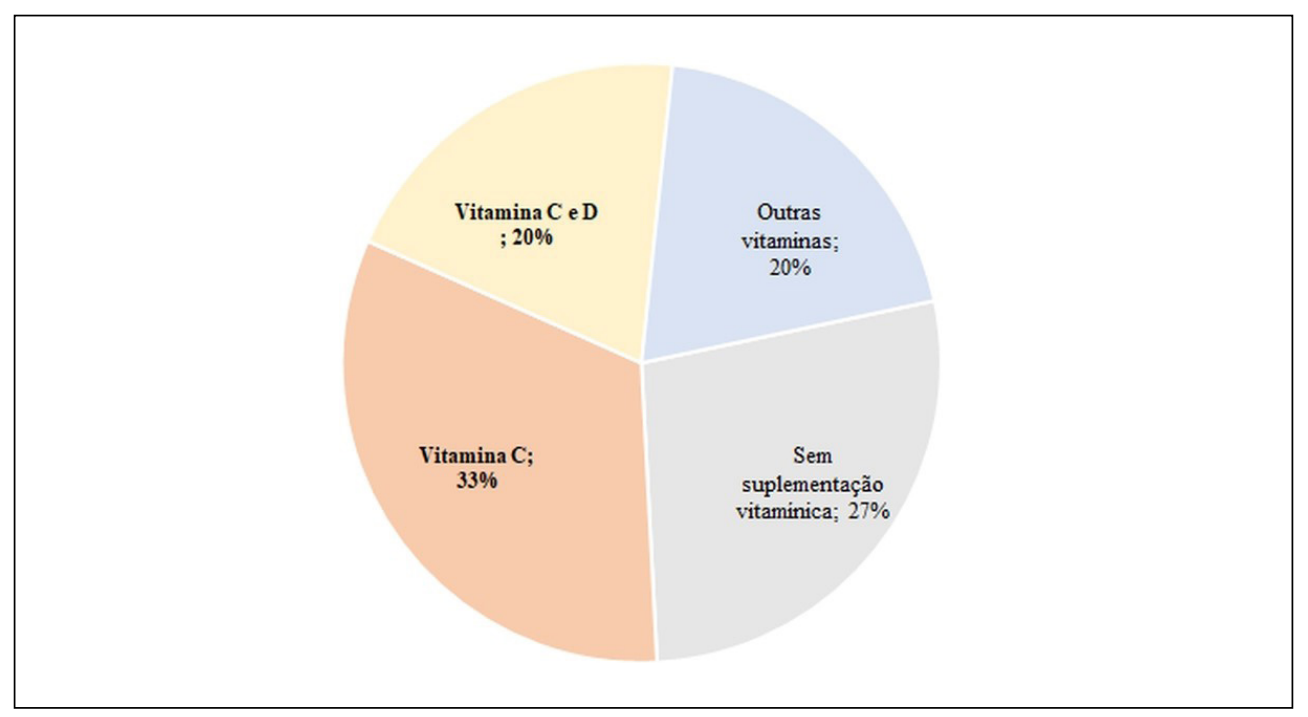

Fonte: Autores.

Com o início da pandemia causada pelo vírus SARS-CoV-2, a vitamina $C$ foi intensamente veiculada como possível amenizador dos sintomas [25]. Segundo o Conselho Federal de Farmácia do Brasil, a venda da vitamina aumentou quase $180 \%$ entre os meses de janeiro a março 2020, se comparado com o mesmo período do ano anterior [26]. Comportamento semelhante ao de 2003, durante o surto de SARS-CoV-1, quando o uso da vitamina $C$ também foi sugerido como tratamento não específico para infecções virais graves do trato respiratório [27].

Dos entrevistados que alegaram fazer uso de suplementos vitamínicos, $28,3 \% \quad(n=189)$ apontaram ter iniciado o uso da vitamina $\mathrm{C}$ devido a pandemia. No passado, esse comportamento foi notado quase que imediatamente em outros países, como quando o consumo de ácido ascórbico quase dobrou no mercado norte-americano em um período de 2 anos, logo após a exposição da pesquisa de Pauling que relacionava o uso da vitamina $\mathrm{C}$ na prevenção e tratamento de resfriado comum em infecções do trato respiratório superior [19].

Um dos questionamentos levantados era se o entrevistado fazia uso concomitante de outros medicamentos, além da vitamina. Em maior número foram citados os anticoncepcionais (57 vezes), seguido dos anti-hipertensivos (53 vezes), outros suplementos alimentares (28 vezes) e antidepressivos (25 vezes). Além dessas classes medicamentosas, também foram citados os anti-inflamatórios esteroides (AIEs) (11 vezes) e não esteroides (AINEs) ( 7 vezes), ansiolíticos ( 7 vezes), anti-histamínicos ( 7 vezes) e antineoplásicos (3 vezes), entre outros. O campo desta resposta era de livre preenchimento, então, apesar da orientação do enunciado, alguns participantes apenas escreveram "sim", mas não apontaram quais medicamentos fazem uso.

Ao contrário do que pensam 43,1\% $(n=563)$ dos entrevistados, que responderam acreditar que o consumo de vitaminas sem acompanhamento médico ou farmacêutico não oferece prejuízo a saúde, a vitamina $\mathrm{C}$ pode interagir em menor ou moderado grau com antineoplásicos (bortezomibe), antiácidos contendo alumínio, contraceptivos orais (etinilestradiol e terapia de reposição hormonal), tetraciclinas, barbitúricos (fenobarbital), AINEs (aspirina e acetaminofeno) e anticoagulantes (varfarina) [28]. 
Além de interações medicamentosas, o consumo da vitamina $\mathrm{C}$ em quantidades maiores que a dose diária recomendada, que varia de $75 \mathrm{mg}$ para mulheres e $90 \mathrm{mg}$ para homens, pode acarretar eventos adversos gastrintestinais como náuseas, vômitos, cólicas abdominais e azia. E, por conta da acidificação da urina, pode elevar os níveis de ácido úrico e oxalato, aumentando risco de cálculos renais $[29,30]$. Neste sentido, a assistência farmacêutica, especialmente, no período de pandemia, é imprescindível para salientar dos benefícios e riscos da suplementação vitamínica [25].

Os outros $56,9 \%(n=742)$ dos participantes reconhecem a importância da supervisão de um profissional. A pesquisa permitia seleção de mais de uma alternativa em relação ao motivo do uso, ainda sim, a maior parte dos participantes indicou ter iniciado o tratamento devido orientação médica $(n=303)$ ou farmacêutica $(n=133)$. Tal resultado pode ser considerado positivo e mostra que, com a difusão de informação nos últimos anos, o reconhecimento e confiança dos pacientes em relação aos profissionais da saúde tem aumentado em relação a assuntos como esse [31].

\section{CONCLUSÃO}

O estudo apresentado mostra que não foram encontradas evidências que liguem de maneira efetiva o consumo da vitamina C com a diminuição da sintomatologia causada pela doença COVID-19, ou indícios que correlacionem a suplementação com a prevenção da doença. Portanto, não é possível presumir que a vitamina C no manejo da COVID-19 terá mesma eficácia que comprovadamente possui na prevenção e tratamento de resfriado comum e de outras comorbidades. Mesmo com a constatação de que os hábitos de consumo de suplementação vitamínica dos entrevistados aumentaram significativamente durante o período da pandemia, é necessário explorar com mais profundidade o papel da vitamina $\mathrm{C}$ e sua relação com a síndrome respiratória causada pela COVID-19.

\section{RFERÊNCIAS BIBLIOGRÁFICAS}

[1] Wu R, Wang L, Kuo HCD, Shannar A, Peter R, Chou PJ, et al. An Update on Current Therapeutic Drugs Treating COVID-19. Curr Pharmacol Reports. 2020;6(3):56-70.

[2] Bilbul M, Paparone P, Kim AM, Mutalik S, Ernst CL. Psychopharmacology of COVID-19. Psychosomatics [Internet] 2020;61(5):411-27. Available from: https://doi.org/10.1016/j. psym.2020.05.006

[3] Beigel JH, Tomashek KM, Dodd LE, Mehta AK, Zingman BS, Kalil AC, et al. Remdesivir for the Treatment of Covid-19 Final Report. N Engl J Med. 2020;383(19):1813-26.

[4] Sanders JM, Monogue ML, Jodlowski TZ, Cutrell JB. Pharmacologic Treatments for Coronavirus Disease 2019 (COVID-19): A Review. JAMA - J Am Med Assoc. 2020;323(18):1824-36.

[5] Morán GAG, Cardona AG, MEJÍA ÓR, Grimaldi DC, Vela SH, Biol $\mathrm{SAB}$, et al. Aspectos bioclínicos y patobiológicos de la vitamina C en la especie humana. Rev CES Med. 2006;20(2):53-72.
[6] Carr AC, Maggini S. Vitamin C and immune function. Nutrients. 2017;9(11):1-25.

[7] Santos JT, Bierhals CC, Feksa LR, Krutzmann MW. Os efeitos da suplementação com vitamina C. Rev Conhecimento Online. 2019; 1:139-63.

[8] Chambial S, Dwivedi S, Shukla KK, John PJ, Sharma P. Vitamin $C$ in disease prevention and cure: An overview. Indian J Clin Biochem. 2013;28(4):314-28.

[9] Tay MZ, Poh CM, Rénia L, MacAry PA, Ng LFP. The trinity of COVID-19: immunity, inflammation and intervention. Nat Rev Immunol [Internet]. 2020;20(6):363-74. Available from: http:// dx.doi.org/10.1038/s41577-020-0311-8

[10] Hemila H. Vitamin C, respiratory infections and the immune system. Trends Immunol. 2003;24(11):579-80.

[11] Zhang L, Liu Y. Potential interventions for novel coronavirus in China: A systematic review. J Med Virol. 2020;92(5):479-90.

[12] Garcia-Diaz DF, Lopez-Legarrea P, Quintero P, Martinez JA. Vitamin $C$ in the treatment and/or prevention of obesity. J Nutr Sci Vitaminol (Tokyo). 2014;60(6):367-79.

[13] Harding AH, Wareham NJ, Bingham SA, Khaw KT, Luben $\mathrm{R}$, Welch $\mathrm{A}$, et al. Plasma vitamin $\mathrm{C}$ level, fruit and vegetable consumption, and the risk of new-onset type 2 diabetes mellitus. Arch Intern Med. 2008;168(14):1493-9.

[14] Iqbal K, Khan A, Khattak MMAK. Biological Significance of Ascorbic Acid (Vitamin C) in Human Health - A Review. Pakistan J Nutr. 2004;3(1):5-13.

[15] Sorice A, Guerriero E, Capone F, Colonna G, Castello G, Costantini S. Ascorbic Acid: Its Role in Immune System and Chronic Inflammation Diseases. Mini-Reviews Med Chem. 2014;14(5):444-52.

[16] Kalantar-Zadeh K, Moore LW. Impact of Nutrition and Diet on COVID-19 Infection and Implications for Kidney Health and Kidney Disease Management. J Ren Nutr. 2020;30(3):179-81.

[17] Ngo B, Van Riper JM, Cantley LC, Yun J. Targeting cancer vulnerabilities with high-dose vitamin C. Nat Rev Cancer [Internet]. 2019;19(5):271-82. Available from: http://dx.doi.org/10.1038/ s41568-019-0135-7

[18] Yang J, Zheng Y, Gou X, Pu K, Chen Z, Guo Q, et al. Prevalence of comorbidities and its effects in coronavirus disease 2019 patients: A systematic review and meta-analysis. Int J Infect Dis. 2020; 94:91-5.

[19] Cerullo G, Negro M, Parimbelli M, Pecoraro M, Perna S, Liguori G, et al. The Long History of Vitamin C: From Prevention of the Common Cold to Potential Aid in the Treatment of COVID-19. Front Immunol. 2020;11:1-16.

[20] de Paiva G da SJ, de Oliveira I de A, Corga JGM, Freitas SM de, Gonçalves ÉCB de A. Avaliação do consumo de compostos bioativos no período da pandemia de Covid-19-uma reflexão. Raízes e Rumos. 2020;8(1):110-30.

[21] Mendes E do N, Fiquene JC, Bessani L, Silva FCM dos S, Máximo NF, Alvarez LS. Hábitos de vida e consumo de alimentos pós pandemia de COVID-19 em São Luís, Maranhão, Brasil. Saúde Coletiva. 2020;10(58):3849-62.

[22] Çimke S, Yıldırım Gürkan D. Determination of interest in 
vitamin use during COVID-19 pandemic using Google Trends data: Infodemiology study. Nutrition. 2021; 85:111138.

[23] Hiedra R, Lo KB, Elbashabsheh M, Gul F, Wright RM, Albano J, et al. The Use of IV vitamin C for patients with COVID-19: a single center observational study. Expert Rev Anti Infect Ther [Internet]. 2020;18(12):1259-61. Available from: https://doi.org/10. 1080/14787210.2020.1794819

[24] Sudre $\mathrm{CH}$, Lee KA, Lochlainn MN, Varsavsky T, Murray B, Graham MS, et al. Symptom clusters in COVID-19: A potential clinical prediction tool from the COVID symptom study app. Sci Adv. 2021;7(12):1-12.

[25] Adams KK, Baker WL, Sobieraj DM. Myth Busters: Dietary Supplements and COVID-19. Ann Pharmacother. 2020;54(8):8206.

[26] Conselho Federal de Farmácia. Levantamento mostra como o medo da Covid-19 impactou venda de medicamentos [Internet]. Comunicação do CFF. 2020. Available from: https:// www.cff.org.br/noticia. php?id=5747

[27] Jesus MN de, Rocha ACFF, Campos SB, Santana TFV, Plácido GR. Vitamina $C$ e a relação com a imunidade e como Agente Preventivo da COVID- 19 (Sars -Cov2). Res Soc Dev. 2021;10(5): e3010514511.

[28] Alagl AS, Bhat SG. Ascorbic acid: new role of an age-old micronutrient in the management of periodontal disease in older adults. Geriatr Gerontol Int. 2015;15(3):241-54.

[29] Jacob RA, Sotoudeh G. Vitamin C function and status in chronic disease. Nutr Clin Care. 2002;5(2):66-74.

[30] Institute of Medicine (US) Panel on Dietary Antioxidants and Related Compounds. Dietary Reference Intakes for Vitamin C, Vitamin E, Selenium, and Carotenoids. Washington (DC): National Academies Press (US). 2000.

[31] Gregory PA, Austin Z. How do patients develop trust in community pharmacists? Res Soc Adm Pharm [Internet]. 2021;17(5):911-20. Available from: https://doi.org/10.1016/j. sapharm.2020.07.023 